JOURNAL OF

SYMPLECTIC GEOMETRY

Volume 1, Number 3, 647-658, 2002

\title{
A remark about Donaldson's construction of symplectic submanifolds
}

\author{
D. Auroux
}

We describe a simplification of Donaldson's arguments for the construction of symplectic hypersurfaces [4] or Lefschetz pencils [5] that makes it possible to avoid any reference to Yomdin's work on the complexity of real algebraic sets.

\section{Introduction.}

Donaldson's construction of symplectic submanifolds [4] is unquestionably one of the major results obtained in the past ten years in symplectic topology. What sets it apart from many of the results obtained during the same period is that it appeals neither to Seiberg-Witten theory, nor to pseudoholomorphic curves; in fact, most of Donaldson's argument is a remarkable succession of elementary observations, combined in a particularly clever way. One ingredient of the proof that does not qualify as elementary, though, is an effective version of Sard's theorem for approximately holomorphic complexvalued functions over a ball in $\mathbb{C}^{n}$ (Theorem 20 in [4]). The proof of this result, which occupies a significant portion of Donaldson's paper ( $\S 4$ and $\S 5$ of [4]), appeals to very subtle considerations about the complexity of real algebraic sets, following ideas of Yomdin [6].

Methods similar to those in [4] were subsequently used to perform various other constructions, leading in particular to Donaldson's result that symplectic manifolds carry structures of symplectic Lefschetz pencils [5], or to the result that symplectic 4-manifolds can be realized as branched coverings of $\mathbb{C P}^{2}[2]$. It was observed in [3] that, whereas Donaldson's construction of submanifolds can be thought of in terms of an estimated transversality result for sections of line bundles, the subsequent constructions can be interpreted in terms of estimated transversality with respect to stratifications in jet bundles.

As remarked at the end of $\S 4$ in [3], the transversality of the $r$-jet of a section to a given submanifold in the bundle of $r$-jets is equivalent to the nonintersection of the $(r+1)$-jet of the section with a certain (possibly singular) 
submanifold of greater codimension in the bundle of $(r+1)$-jets. This is of particular interest because the effective Sard theorem for approximately holomorphic functions from $\mathbb{C}^{n}$ to $\mathbb{C}^{m}$ admits a conceptually much easier proof in the case where $m>n$ [2]. In the case of the construction of symplectic submanifolds, the formalism of jet bundles can be completely eliminated from the presentation; the purpose of this note is to present the resulting simplified argument for Donaldson's result ( $\S 2-3)$. We also observe (see $\S 4$ ) that a similar simplification is possible for the higher-rank local result required for the construction of symplectic Lefschetz pencils [5].

\section{Overview of Donaldson's argument.}

We first review Donaldson's construction of symplectic submanifolds [4], using the terminology and notations of [2]. Let $\left(X^{2 n}, \omega\right)$ be a compact symplectic manifold, and assume that the cohomology class $\frac{1}{2 \pi}[\omega]$ is integral. Endow $X$ with an $\omega$-compatible almost-complex structure $J$ and the corresponding Riemannian metric $g=\omega(\cdot, J \cdot)$. Consider a Hermitian line bundle $L$ over $X$ such that $c_{1}(L)=\frac{1}{2 \pi}[\omega]$, equipped with a Hermitian connection $\nabla$ having curvature $-i \omega$. The almost-complex structure $J$ induces a splitting of the connection into $\nabla=\partial+\bar{\partial}$. We are interested in approximately holomorphic sections of the line bundles $L^{\otimes k}(k \gg 0)$ satisfying a certain estimated transversality property: indeed, if we can find a section $s$ such that $|\bar{\partial} s| \ll|\partial s|$ at every point where $s$ vanishes, then the zero set of $s$ is automatically a smooth symplectic submanifold in $X$ (cf. e.g. Proposition 3 of [4]). The philosophical justification of the construction is that, as the twisting parameter $k$ increases, one starts probing the geometry of $X$ at very small scales, where the effects due to the non-integrability of $J$ become negligible. This phenomenon is due to the curvature $-i k \omega$ of the connection on $L^{\otimes k}$, and leads us to work with a rescaled metric $g_{k}=k g$ (the metric induced by $J$ and $k \omega$ ).

Let $\left(s_{k}\right)_{k \gg 0}$ be a sequence of sections of Hermitian vector bundles $E_{k}$ equipped with Hermitian connections over $X$. We make the following definitions:

Definition 1. The sections $s_{k}$ are asymptotically holomorphic if there exist constants $\left(C_{p}\right)_{p \in \mathbb{N}}$ such that, for all $k$ and at every point of $X,\left|s_{k}\right| \leq C_{0}$, $\left|\nabla^{p} s_{k}\right|_{g_{k}} \leq C_{p}$ and $\left|\nabla^{p-1} \bar{\partial} s_{k}\right|_{g_{k}} \leq C_{p} k^{-1 / 2}$ for all $p \geq 1$.

Definition 2. The sections $s_{k}$ are uniformly transverse to 0 if there exists a constant $\eta>0$ independent of $k$ such that the sections $s_{k}$ are $\eta$-transverse 
to 0 , i.e. if at any point $x \in X$ where $\left|s_{k}(x)\right|<\eta$, the linear map $\nabla s_{k}(x)$ : $T_{x} X \rightarrow\left(E_{k}\right)_{x}$ is surjective and has a right inverse of norm less than $\eta^{-1}$ w.r.t. the metric $g_{k}$.

When $\operatorname{rank}\left(E_{k}\right)>n$, uniform transversality means that $\left|s_{k}(x)\right| \geq \eta$ at every point of $X$; on the other hand, when $E_{k}$ is a line bundle and the sections $s_{k}$ are asymptotically holomorphic, uniform transversality can be rephrased as a uniform lower bound on $\left|\partial s_{k}\right|$ at all points where $\left|s_{k}\right|<$ $\eta$ (which by the above observation is enough to ensure the symplecticity of $s_{k}^{-1}(0)$ for large $k$ ). With this terminology, Donaldson's result can be reformulated as follows (cf. Theorem 5 of [4]):

Theorem 1. For large values of $k$, the line bundles $L^{\otimes k}$ admit sections $s_{k}$ that are asymptotically holomorphic and uniformly transverse to 0.

The proof of Theorem 1 starts with a couple of preliminary lemmas about the existence of approximately holomorphic rescaled Darboux coordinates on $X$ and of large families of well-concentrated asymptotically holomorphic sections of $L^{\otimes k}$.

Lemma 1. There exists a constant $c>0$ such that near any point $x \in$ $X$, for any integer $k$, there exist local complex Darboux coordinates $z_{k}=$ $\left(z_{k}^{1}, \ldots, z_{k}^{n}\right):(X, x) \rightarrow\left(\mathbb{C}^{n}, 0\right)$ for the symplectic structure $k \omega$, such that the following estimates hold uniformly in $x$ and $k$ at every point of the ball $B_{g_{k}}(x, c \sqrt{k}):\left|z_{k}(y)\right|=O\left(\operatorname{dist}_{g_{k}}(x, y)\right),\left|\bar{\partial} z_{k}(y)\right|_{g_{k}}=O\left(k^{-1 / 2} \operatorname{dist}_{g_{k}}(x, y)\right)$, $\left|\nabla^{r} \bar{\partial} z_{k}\right|_{g_{k}}=O\left(k^{-1 / 2}\right),\left|\nabla^{r} z_{k}\right|_{g_{k}}=O(1) \forall r \geq 1$; and denoting by $\psi_{k}$ : $\left(\mathbb{C}^{n}, 0\right) \rightarrow(X, x)$ the inverse map, the estimates $\left|\bar{\partial} \psi_{k}(z)\right|_{g_{k}}=O\left(k^{-1 / 2}|z|\right)$, $\left|\nabla^{r} \bar{\partial} \psi_{k}\right|_{g_{k}}=O\left(k^{-1 / 2}\right)$ and $\left|\nabla^{r} \psi_{k}\right|_{g_{k}}=O(1)$ hold $\forall r \geq 1$ at every point of the ball $B_{\mathbb{C}^{n}}(0, c \sqrt{k})$, where $\bar{\partial} \psi_{k}$ is defined with respect to the almost-complex structure $J$ on $X$ and the standard complex structure on $\mathbb{C}^{2}$.

Lemma 1 is identical to Lemma 3 of [2], or to the discussion on pp. 674675 of [4] if one keeps track carefully of the available estimates; the idea is simply to start with usual Darboux coordinates for $\omega$, compose them with a linear transformation to ensure holomorphicity at the origin, and then rescale them by a factor of $\sqrt{k}$.

Definition 3. A section $s$ of $E_{k}$ has Gaussian decay in $C^{r}$ norm away from a point $x \in X$ if there exist a polynomial $P$ and a constant $\lambda>0$ such that for all $y \in X,|s(y)|,|\nabla s(y)|_{g_{k}}, \ldots,\left|\nabla^{r} s(y)\right|_{g_{k}}$ are all bounded by $P(d(x, y)) \exp \left(-\lambda d(x, y)^{2}\right)$, where $d(.,$.$) is the distance induced by g_{k}$. The 
decay properties of a family of sections are said to be uniform if $P$ and $\lambda$ can be chosen independently of $k$ and of the point $x$ at which decay occurs for a given section.

Lemma 2. Given any point $x \in X$, for all large enough $k$, there exist asymptotically holomorphic sections $s_{k, x}^{\mathrm{ref}}$ of $L^{\otimes k}$ over $X$, such that $\left|s_{k, x}^{\mathrm{ref}}\right| \geq c_{0}$ at every point of the ball of $g_{k}$-radius 1 centered at $x$, for some universal constant $c_{0}>0$, and such that the sections $s_{k, x}^{\text {ref }}$ have uniform Gaussian decay away from $x$ in $C^{2}$ norm.

Lemma 2 is essentially Proposition 11 of [4]. Considering a local trivialization of $L^{\otimes k}$ where the connection 1-form is $\frac{1}{4} \sum\left(z_{k}^{j} d \bar{z}_{k}^{j}-\bar{z}_{k}^{j} d z_{k}^{j}\right)$, the sections $s_{k, x}^{\text {ref }}$ are constructed by multiplication of the function $\exp \left(-\left|z_{k}\right|^{2} / 4\right)$ by a suitable cut-off function at distance $k^{1 / 6}$ from the origin.

The central ingredient is the following result about the near-critical sets of approximately holomorphic functions (used in the special case $m=1$ ):

Proposition 1. Let $f$ be a function defined over the ball $B^{+}$of radius $\frac{11}{10}$ in $\mathbb{C}^{n}$ with values in $\mathbb{C}^{m}$. Let $\delta$ be a constant with $0<\delta<\frac{1}{4}$, and let $\eta=\delta \log \left(\delta^{-1}\right)^{-p}$ where $p$ is a fixed integer depending only on $n$ and $m$. Assume that $f$ satisfies the bounds $|f|_{C^{0}\left(B^{+}\right)} \leq 1$ and $|\bar{\partial} f|_{C^{1}\left(B^{+}\right)} \leq \eta$. Then there exists $w \in \mathbb{C}^{m}$ with $|w| \leq \delta$ such that $f-w$ is $\eta$-transverse to 0 over the interior ball $B$ of radius 1 .

The case $m=1$ is Theorem 20 of [4]; the comparatively much easier case $m>n$ is Proposition 2 of [2]; the general case is proved in [5]. In all cases the proof begins with an approximation of $f$ first by a holomorphic function (using general elliptic theory), then by a polynomial $g$ of degree $O\left(\log \left(\eta^{-1}\right)\right)$ (by truncating the power series expansion at the origin). The proof in the case $m=1$ then appeals to a rather sophisticated result on the complexity of real algebraic sets to control the size of the set of points where $\partial g$ is small (the near-critical points) [4]. Meanwhile, in the case $m>n$, since we only have to find $w$ such that $|f-w| \geq \eta$ at every point of $B$, it is sufficient to observe that the image of the polynomial map $g$ is contained in a complex algebraic hypersurface $H$ in $\mathbb{C}^{m}$; the result then follows from a standard result about the volume of a tubular neighborhood of $H$, which can be estimated using an explicit bound on the degree of $H$ [2].

Given asymptotically holomorphic sections $s_{k}$ of $L^{\otimes k}$ and a point $x \in X$, one can apply Proposition 1 to the complex-valued functions $f_{k}=s_{k} / s_{k, x}^{\mathrm{ref}}$ (defined over a neighborhood of $x$ ) in order to find constants $w_{k}$ such that the functions $f_{k}-w_{k}$ are uniformly transverse to 0 near $x$; multiplying by 
$s_{k, x}^{\text {ref }}$, it follows that the sections $s_{k}-w_{k} s_{k, x}^{\text {ref }}$ are uniformly transverse to 0 near $x$. Therefore, we have:

Proposition 2. There exist constants $c, c^{\prime}, p, \delta_{0}>0$ such that, given a real number $\delta \in\left(0, \delta_{0}\right)$, a sequence of asymptotically holomorphic sections $s_{k}$ of $L^{\otimes k}$ and a point $x \in X$, for large enough $k$ there exist asymptotically holomorphic sections $\tau_{k, x}$ of $L^{\otimes k}$ with the following properties: (a) $\left|\tau_{k, x}\right|_{C^{1}, g_{k}}<\delta$, (b) the sections $\frac{1}{\delta} \tau_{k, x}$ have uniform Gaussian decay away from $x$ in $C^{1}$ norm, and (c) the sections $s_{k}+\tau_{k, x}$ are $\eta$-transverse to 0 at every point of the ball $B_{g_{k}}(x, c)$, with $\eta=c^{\prime} \delta \log \left(\delta^{-1}\right)^{-p}$.

This result lets us achieve estimated transversality over a small ball in $X$ by adding to $s_{k}$ a small well-concentrated perturbation. Uniform transversality over the entire manifold $X$ is achieved by proceeding iteratively, adding successive perturbations to the sections in order to obtain transversality properties over larger and larger subsets of $X$. The key observation is that estimated transversality is an open property (preserved under $C^{1}$-small perturbations). Since the transversality estimate decreases after each perturbation, it is important to obtain global uniform transversality after a number of steps that remains bounded independently of $k$; this is made possible by the uniform decay properties of the perturbations, using a beautiful observation of Donaldson. The reader is referred to $\S 3$ of [4] or to Proposition 3 of [2] for details.

\section{The simplified argument.}

Keeping the same general strategy, the proof of Theorem 1 can be simplified by appealing to a result weaker than Proposition 1, namely the following statement:

Proposition 3. Let $f$ be a function defined over the ball $B^{+}$of radius $\frac{11}{10}$ in $\mathbb{C}^{n}$ with values in $\mathbb{C}$. Let $\delta$ be a constant with $0<\delta<\frac{1}{4}$, and let $\eta=\delta \log \left(\delta^{-1}\right)^{-p^{\prime}}$ where $p^{\prime}$ is a fixed integer depending only on $n$. Assume that $f$ satisfies the bounds $|f|_{C^{1}\left(B^{+}\right)} \leq 1$ and $|\bar{\partial} f|_{C^{2}\left(B^{+}\right)} \leq \eta$. Then there exists $w=\left(w_{0}, w_{1}, \ldots, w_{n}\right) \in \mathbb{C}^{n+1}$ with $|w| \leq \delta$ such that the function $f-w_{0}-\sum w_{i} z_{i}$ is $\eta$-transverse to 0 over the interior ball $B$ of radius 1 .

Proof. Let $g=\left(g_{0}, \ldots, g_{n}\right): B^{+} \rightarrow \mathbb{C}^{n+1}$ be the function defined by $g_{i}=$ $\partial f / \partial z_{i}$ for $1 \leq i \leq n$ and $g_{0}=f-\sum_{i=1}^{n} z_{i} g_{i}$. The bounds on $f$ immediately imply that $|g|_{C^{0}\left(B^{+}\right)} \leq C_{n}$ and $|\bar{\partial} g|_{C^{1}\left(B^{+}\right)} \leq C_{n} \eta$, for some constant $C_{n}$ 
depending only on the dimension. We can safely choose the constant $p^{\prime}$ appearing in the definition of $\eta$ to be larger than the constant $p$ appearing in Proposition 1. Therefore we can apply Proposition 1 in its easy version $(m=n+1)$ to the function $g$, after scaling down by the constant factor $C_{n}$. This gives us a constant $w=\left(w_{0}, \ldots, w_{n}\right) \in \mathbb{C}^{n+1}$, bounded by $\delta$, and such that $|g-w| \geq \alpha$ at every point of $B$, where $\alpha=\delta \log \left(\left(\delta / C_{n}\right)^{-1}\right)^{-p}$.

Define $\tilde{f}=f-w_{0}-\sum w_{i} z_{i}$ and $\tilde{g}=g-w$, and observe that $\partial \tilde{f} / \partial z_{i}=\tilde{g}_{i}$ for $1 \leq i \leq n$ and $\tilde{f}-\sum_{i=1}^{n} z_{i} \tilde{g}_{i}=\tilde{g}_{0}$. Let $z \in B$ be a point where $|\partial \tilde{f}|<\frac{1}{4} \alpha$. Since $\partial \tilde{f} / \partial z_{i}=\tilde{g}_{i}$, and since $|\tilde{g}(z)| \geq \alpha$ by construction, we have the inequality $\left|\tilde{g}_{0}(z)\right|>\frac{3}{4} \alpha$. However, $\left|\tilde{f}(z)-\tilde{g}_{0}(z)\right|=\left|\sum z_{i} \tilde{g}_{i}(z)\right| \leq|z||\partial \tilde{f}(z)|<\frac{1}{4} \alpha$ (recall that $z$ belongs to the unit ball). Therefore $|\tilde{f}(z)|>\frac{1}{2} \alpha$.

Conversely, at any point $z \in B$ where $|\tilde{f}| \leq \frac{1}{2} \alpha$ we must have $|\bar{\partial} \tilde{f}(z)| \geq \frac{1}{4} \alpha$. However, because of the bound on $\bar{\partial} \tilde{f}=\bar{\partial} f$, if we assume that $\eta<\frac{1}{8} \alpha$ then this inequality implies that $\nabla \tilde{f}(z)$ is surjective and admits a right inverse of norm at most $\left(\frac{1}{8} \alpha\right)^{-1}$. Hence we conclude from the previous discussion that $\tilde{f}$ is $\frac{1}{8} \alpha$-transverse to 0 over $B$. Finally, we observe that, because $\delta<\frac{1}{4}$, if the constant $p^{\prime}$ is chosen large enough then $\eta=\delta \log \left(\delta^{-1}\right)^{-p^{\prime}}<\frac{1}{8} \alpha=\frac{1}{8} \delta \log \left(\left(\delta / C_{n}\right)^{-1}\right)^{-p}$, so that $\tilde{f}$ is $\eta$-transverse to 0 over $B$.

Although it is weaker, Proposition 3 is in practice interchangeable with the case $m=1$ of Proposition 1, in particular for the purpose of proving Proposition 2.

Proof of Proposition 2. We use the same argument as Donaldson [4]: we work in approximately holomorphic Darboux coordinates on a neighborhood of the given point $x$, using Lemma 1 . Using the sections $s_{k, x}^{\text {ref }}$ given by Lemma 2 to define local trivializations of $L^{\otimes k}$, the sections $s_{k}$ can be identified with complex-valued functions $f_{k}=s_{k} / s_{k, x}^{\text {ref }}$. The estimates on $s_{k}$

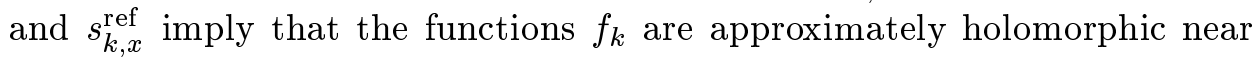
the origin (in particular $\left|\bar{\partial} f_{k}\right|_{C^{2}}=O\left(k^{-1 / 2}\right)$ ); after a suitable rescaling of the coordinates and of the functions by uniform constant factors, we can assume additionally that $\left|f_{k}\right|_{C^{1}} \leq 1$ near the origin, and that the estimates hold over a neighborhood of the origin that contains the ball $B^{+}$. Therefore, the assumptions of Proposition 3 are satisfied provided that $k$ is sufficiently large to ensure that $k^{-1 / 2} \ll \eta$.

By Proposition 3 , we can find $w_{k}=\left(w_{k, 0}, \ldots, w_{k, n}\right) \in \mathbb{C}^{n+1}$, with $\left|w_{k}\right| \leq$ $\delta$, such that $\tilde{f}_{k}=f_{k}-w_{k, 0}-\sum w_{k, i} z_{i}$ is $\gamma$-transverse to 0 over the unit 
ball, where $\gamma=\delta \log \left(\delta^{-1}\right)^{-p^{\prime}}$. Define $\tau_{k, x}=-w_{k, 0} s_{k, x}^{\mathrm{ref}}-\sum w_{k, i} z_{k}^{i} s_{k, x}^{\mathrm{ref}}$. The estimates on $z_{k}^{i}$ from Lemma 1 and on $s_{k, x}^{\text {ref }}$ from Lemma 2 imply that the sections $z_{k, i} s_{k, x}^{\text {ref }}$ of $L^{\otimes k}$ are asymptotically holomorphic and have uniform Gaussian decay away from $x$. Therefore, it is easy to check that the sections $\frac{1}{\delta} \tau_{k, x}$ are asymptotically holomorphic and have uniform Gaussian decay. Moreover, because there exist uniform bounds on $s_{k, x}^{\text {ref }}$ and $z_{k}^{i} s_{k, x}^{\text {ref }}$, one easily checks that $\left|\tau_{k, x}\right|_{C^{1}, g_{k}}$ is bounded by some constant multiple of $\delta$; decreasing the required bound on $\left|w_{k}\right|$, we can assume that the constant is equal to 1 , to the expense of inserting a constant factor in the above expression for $\gamma$. Finally, observing that $s_{k}+\tau_{k, x}=\tilde{f}_{k} s_{k, x}^{\text {ref }}$ over a neighborhood of $x$, it is straightforward to check that the $\gamma$-transversality to 0 of $\tilde{f}_{k}$ and the lower bound satisfied by $s_{k, x}^{\text {ref }}$ imply a uniform transversality property of the desired form for the section $s_{k}+\tau_{k, x}$.

Remark 1. Proposition 3 also admits a version for one-parameter families of functions: given functions $f_{t}: B^{+} \rightarrow \mathbb{C}$ depending continuously on a parameter $t \in[0,1]$ and satisfying the assumptions of Proposition 3 for all values of $t$, we can find constants $w_{t} \in \mathbb{C}^{n+1}$, depending continuously on $t$, such that the conclusion holds for all values of $t$. This is because the auxiliary functions $g_{t}: B^{+} \rightarrow \mathbb{C}^{n+1}$ introduced in the proof also depend continuously on $t$, which allows us to appeal to the one-parameter version of Proposition 1 (cf. e.g. Proposition 2 of [2]). We can therefore simplify the argument proving the asymptotic uniqueness of the constructed submanifolds [1] in the same manner as the construction itself.

Remark 2. The idea behind the modified argument can be interpreted as follows in terms of 1-jets of sections: let $\mathcal{J}^{1} L^{\otimes k}=L^{\otimes k} \oplus\left(T^{*} X^{1,0} \otimes L^{\otimes k}\right)$, and define the 1-jet of a section $s_{k} \in \Gamma\left(L^{\otimes k}\right)$ as $j^{1} s_{k}=\left(s_{k}, \partial s_{k}\right) \in \Gamma\left(\mathcal{J}^{1} L^{\otimes k}\right)$. The jet bundles carry natural Hermitian metrics (induced by those on $L^{\otimes k}$ and the metrics $g_{k}$ on the cotangent bundle), and natural Hermitian connections for which the 1-jets of asymptotically holomorphic sections of $L^{\otimes k}$ are asymptotically holomorphic sections of $\mathcal{J}^{1} L^{\otimes k}$. It is worth noting that the natural connection on $\mathcal{J}^{1} L^{\otimes k}$ is not the connection $\nabla$ induced by the connection on $L^{\otimes k}$ and the Levi-Civita connection, because $\bar{\partial}^{\nabla}\left(s_{k}, \partial s_{k}\right)=\left(\bar{\partial} s_{k}, \bar{\partial} \partial s_{k}\right)$ differs from $\left(\bar{\partial} s_{k},-\partial \bar{\partial} s_{k}\right)$ (which is bounded by $O\left(k^{-1 / 2}\right)$ ) by the curvature term $-i k \omega s_{k}$. Therefore, we must instead work with the Hermitian connection $\tilde{\nabla}$ characterized by the formula $\bar{\partial}^{\tilde{\nabla}}\left(\sigma^{0}, \sigma^{1}\right)=\bar{\partial}^{\nabla}\left(\sigma^{0}, \sigma^{1}\right)+\left(0, i k \omega \sigma^{0}\right)$, where $\omega$ is viewed as a $(0,1)$-form with values in $T^{*} X^{1,0}$. 
Observe that the 1 -jets $j^{1} \sigma_{k, x, 0}, \ldots, j^{1} \sigma_{k, x, n}$, where $\sigma_{k, x, 0}=s_{k, x}^{\text {ref }}$ and $\sigma_{k, x, i}=z_{k}^{i} s_{k, x}^{\text {ref }}$ for $1 \leq i \leq n$, are asymptotically holomorphic sections of $\mathcal{J}^{1} L^{\otimes k}$, with uniform Gaussian decay away from $x$, which form a local frame of the jet bundle over a neighborhood of $x$. Therefore, given asymptotically holomorphic sections $s_{k}$ and a point $x \in X$, there exist local complex-valued functions $g_{k, 0}, \ldots, g_{k, n}$ such that $j^{1} s_{k}=\sum g_{k, i} j^{1} \sigma_{k, x, i}$. Moreover, remark that a section of $L^{\otimes k}$ is uniformly transverse to 0 if and only if its 1-jet satisfies a uniform lower bound. Therefore, our argument actually amounts to a local perturbation of $j^{1} s_{k}$, using the given local frame $\left\{j^{1} \sigma_{k, x, i}\right\}$, in order to bound it away from 0 ; because the rank of the jet bundle is $n+1>$ $n$, the easy version of Proposition 1 is sufficient for that purpose. The curious reader is referred to [3] for a more detailed discussion of estimated transversality using the formalism of jet bundles.

\section{The higher-rank local result.}

We now formulate and prove an analogue of Proposition 3 for functions with values in $\mathbb{C}^{m}(m \leq n)$; as in the case $m=1$, the statement differs from Proposition 1 by allowing the extra freedom of affine perturbations rather than restricting oneself to constants.

Proposition 4. Let $f$ be a function defined over the ball $B^{+}$of radius $\frac{11}{10}$ in $\mathbb{C}^{n}$ with values in $\mathbb{C}^{m}, m \leq n$. Let $\delta$ be a constant with $0<\delta<\frac{1}{4}$, and let $\eta=\delta \log \left(\delta^{-1}\right)^{-p^{\prime}}$ where $p^{\prime}$ is a fixed integer depending only on $m$ and $n$. Assume that $f$ satisfies the bounds $|f|_{C^{0}\left(B^{+}\right)} \leq 1$ and $|\bar{\partial} f|_{C^{1}\left(B^{+}\right)} \leq \eta$. Then there exists $w=\left(w_{0}, w_{1}, \ldots, w_{n}\right) \in \mathbb{C}^{m(n+1)}$ (each $w_{i}$ is an element of $\left.\mathbb{C}^{m}\right)$ with $|w| \leq \delta$ such that the function $f-w_{0}-\sum w_{i} z_{i}$ is $\eta$-transverse to 0 over the interior ball $B$ of radius 1 .

Moreover, given a one-parameter family of functions $f_{t}: B^{+} \rightarrow \mathbb{C}$ depending continuously on a parameter $t \in[0,1]$ and satisfying the above assumptions for all $t$, we can find constants $w_{t} \in \mathbb{C}^{m(n+1)}$, depending continuously on $t$, such that the conclusion holds for all values of $t$.

This statement is essentially interchangeable with Proposition 1 for all practical applications, and in particular the case $m=n$ allows us to simplify noticeably the argument for Donaldson's construction of symplectic Lefschetz pencils [5]. Indeed, the main problem to be solved is the following: given pairs of asymptotically holomorphic sections $\left(s_{k}^{0}, s_{k}^{1}\right)$ of $L^{\otimes k}$, defining $\mathbb{C P}^{1}$-valued maps $f_{k}=\left[s_{k}^{0}: s_{k}^{1}\right]$ away from the base loci, one must perturb them so that the differentials $\partial f_{k}$ (which are sections of rank $n$ 
vector bundles) become uniformly transverse to 0 . This ensures the nondegeneracy of the singular points of the pencil. The manner in which the problem reduces to the $m=n$ case of Proposition 1 is explained in detail in [5], and the reduction to Proposition 4 is essentially identical except that the resulting perturbations of $\left(s_{k}^{0}, s_{k}^{1}\right)$ are products of $s_{k, x}^{\text {ref }}$ by quadratic (rather than linear) polynomials.

Proof. Although for technical reasons we cannot use directly the case $m>n$ of Proposition 1, the argument presents many similarities with $\S 2.3$ of [2]; we accordingly skip the details whenever the two arguments parallel each other in an obvious manner. As in the case of Proposition 1, we first use the bounds on $f$ to find an approximation by a polynomial $h: \mathbb{C}^{n} \rightarrow \mathbb{C}^{m}$ of degree $d=O\left(\log \left(\eta^{-1}\right)\right)$ such that $|h-f|_{C^{1}(B)} \leq c \eta$ for some constant $c$ (see Lemmas 27 and 28 of [4]). Observe that, if we can perturb $h$ by less than $\delta$ to make it $(c+1) \eta$-transverse to 0 over $B$, then because transversality is an open property the desired result on $f$ will follow immediately. So we are reduced to the case of a polynomial function $h=\left(h^{1}, \ldots, h^{m}\right)$ of degree $d=O\left(\log \left(\eta^{-1}\right)\right)$.

If $w=\left(w_{0}, \ldots, w_{n}\right)$ is a vector in $\mathbb{C}^{m(n+1)}$, denote by $\left(w_{i}^{j}\right)_{1 \leq j \leq m}$ the components of $w_{i}$, and let $\vec{w}=\left(w_{1}, \ldots, w_{n}\right) \in \mathbb{C}^{m \times n}$. The set of choices to be avoided for $w$ is

$S=\left\{w \in \mathbb{C}^{m(n+1)}, \exists z \in B\right.$ s.t. $\left.h(z)-w_{0}-\sum w_{i} z_{i}=0, \bigwedge^{m}(\partial h(z)-\vec{w})=0\right\}$.

Indeed, observe that $h-w_{0}-\sum w_{i} z_{i}$ is transverse to 0 over $B$ (without any estimate) if and only if $w \notin S$. We now define a polynomial function $g: \mathbb{C}^{N-1} \rightarrow \mathbb{C}^{N}$, where $N=m(n+1)$, which parametrizes a dense subset of $S$. Given elements $z=\left(z_{i}\right)_{1 \leq i \leq n} \in \mathbb{C}^{n}, \theta=\left(\theta_{i}^{j}\right)_{1 \leq i \leq n, 1 \leq j \leq m-1} \in \mathbb{C}^{(m-1) n}$ and $\lambda=\left(\lambda_{j}\right)_{1 \leq j \leq m-1} \in \mathbb{C}^{m-1}$, we define $g(z, \theta, \lambda) \in \mathbb{C}^{m(n+1)}$ by the formulas

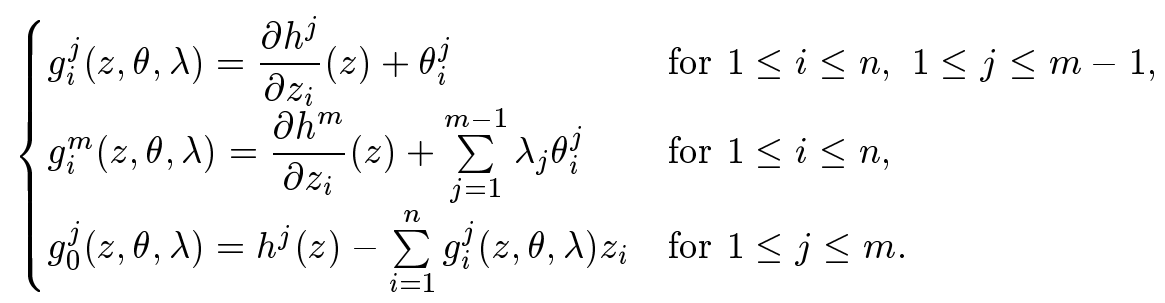

One easily checks that the image by $g$ of the subset $\left\{(z, \theta, \lambda) \in \mathbb{C}^{N-1}\right.$, $z \in B\}$ is contained in $S$, in which it is a dense subset. Observe that $g$ is a polynomial map with the same degree $d$ as $h$ (provided that $d \geq 2$ ). Therefore, the image $g\left(\mathbb{C}^{N-1}\right)$ is contained in an algebraic surface $H \subset$ 
$\mathbb{C}^{N}$, of degree at most $D=N d^{N-1}$. Indeed, denoting by $E$ the space of polynomials of degree at most $D$ in $N$ variables and by $E^{\prime}$ the space of polynomials of degree at most $d D$ in $N-1$ variables, we have $\operatorname{dim} E=$ $\left(\begin{array}{c}D+N \\ N\end{array}\right)>\left(\begin{array}{c}d D+N-1 \\ N-1\end{array}\right)=\operatorname{dim} E^{\prime}$, so that the map from $E$ to $E^{\prime}$ defined by $P \mapsto P \circ g$ cannot be injective, and a non-zero element of its kernel provides an equation for the hypersurface $H$ (see $\S 2.3$ of [2] for details).

Since $g\left(B \times \mathbb{C}^{(m-1) n} \times \mathbb{C}^{m-1}\right)$ is dense in $S$, we conclude that $S \subset H$. From this point on, the argument is very similar to $\S 2.3$ of [2], to which the reader is referred for details. Standard results on complex algebraic hypersurfaces, essentially amounting to the well-known monotonicity formula, allow us to bound the size of $S$ and of its tubular neighborhoods (cf. e.g. Lemma 4 of [2]). In particular, denoting by $\bar{B}$ the ball of radius $\delta$ centered at the origin in $\mathbb{C}^{N}$ and by $V_{0}$ the volume of the unit ball in dimension $2 N-2$, we have $\operatorname{vol}_{2 N-2}(H \cap \bar{B}) \leq D V_{0} \delta^{2 N-2}$, while given any point $x \in H$ we have $v l_{2 N-2}(H \cap B(x, \eta)) \geq V_{0} \eta^{2 N-2}$. Therefore, choosing a suitable covering of $\bar{B}$ by balls of radius $\eta$, one can show that $H \cap \bar{B}$ is contained in the union of $M=C D \delta^{2 N-2} \eta^{-(2 N-2)}$ balls of radius $\eta$, where $C$ is a constant depending only on $N$. As a consequence, the neighborhood $Z=\left\{w \in \mathbb{C}^{N},|w| \leq \delta\right.$, $\operatorname{dist}(w, S) \leq(3 c+3) \eta\}$ is contained in the union of $M$ balls of radius $(3 c+4) \eta$.

A simple comparison of the volumes implies that, if the constant $p^{\prime}$ is chosen suitably large, then the volume of $Z$ is much smaller than that of the ball $\bar{B}$, and therefore $\bar{B}-Z$ is not empty, i.e. $\bar{B}$ contains an element $w$ which lies at distance more than $(3 c+3) \eta$ from $S$. Moreover, using a standard isoperimetric inequality we can show that $\bar{B}-Z$ contains a unique large connected component; it follows that, in the case where the data depends continuously on a parameter $t \in[0,1]$, the subset $\bigsqcup\{t\} \times\left(\bar{B}-Z_{t}\right) \subset[0,1] \times \bar{B}$ contains a preferred large connected component, in which we can choose elements $w_{t}$ depending continuously on $t$.

To complete the proof of Proposition 4, we only need to show that, if $w \in \bar{B}$ lies at distance more than $(3 c+3) \eta$ from $S$, then $\tilde{f}=f-w_{0}-\sum w_{i} z_{i}$ is $\eta$-transverse to 0 over $B$. In fact, it is sufficient to show that $\tilde{h}=h-$ $w_{0}-\sum w_{i} z_{i}$ is $(c+1) \eta$-transverse to 0 over $B$, because $|\tilde{h}-\tilde{f}|_{C^{1}(B)}=$ $|h-f|_{C^{1}(B)} \leq c \eta$ and transversality is an open property. We conclude using the following lemma:

Lemma 3. If $w$ lies at distance more than $3 \alpha$ from $S$ for some constant $\alpha>0$, then $\tilde{h}=h-w_{0}-\sum w_{i} z_{i}$ is $\alpha$-transverse to 0 over $B$.

To prove Lemma 3, we first provide an alternative definition of $\alpha$-transversality: 
Lemma 4. Let $L: E \rightarrow F$ be a linear map between Hermitian complex vector spaces, and choose a constant $\alpha>0$. The two following properties are equivalent:

(i) $L$ is surjective and has a right inverse $R: F \rightarrow E$ of norm at most $\alpha^{-1}$,

(ii) for every unit vector $v$ in $F$, the component $\langle v, L\rangle=v^{*} L$ of $L$ along $v$ is a linear form on $E$ such that $\left|v^{*} L\right| \geq \alpha$.

Proof. If $(i)$ holds, then given any unit vector $v \in F$, the vector $u=R v$ is such that $|u| \leq \alpha^{-1}$ and $\langle v, L u\rangle=|v|^{2}=1$. Therefore the linear form $\langle v, L\rangle$ has norm at least $\alpha$, and (ii) holds.

Conversely, assume (ii) holds. Then for any $v \in F$ we have $\left|v^{*} L\right| \geq \alpha|v|$, i.e. $v^{*} L L^{*} v \geq \alpha^{2}|v|^{2}$. Therefore, the Hermitian endomorphism $L L^{*}$ of $F$ is positive definite and has eigenvalues $\geq \alpha^{2}$. It follows that it admits an inverse $U=\left(L L^{*}\right)^{-1}$ of operator norm at most $\alpha^{-2}$. We have $L L^{*} U=\mathrm{Id}$, and $\left|L^{*} U v\right|^{2}=\left\langle v, U L L^{*} U v\right\rangle=\langle v, U v\rangle \leq \alpha^{-2}|v|^{2}$, so that $R=L^{*} U$ is a right inverse of norm at most $\alpha^{-1}$.

Proof of Lemma 3. Assume that $\tilde{h}$ is not $\alpha$-transverse to 0 over $B$ : using the definition and Lemma 4 , there exists a point $z \in B$ and a unit vector $v \in \mathbb{C}^{m}$ such that $|\tilde{h}(z)|<\alpha$ and $|\langle v, \partial \tilde{h}(z)\rangle|<\alpha$. Let $u=\left(u_{0}, u_{1}, \ldots, u_{n}\right) \in$ $\mathbb{C}^{m(n+1)}$ be such that $u_{i}=\left\langle v, \partial \tilde{h} / \partial z_{i}\right\rangle v$ and $u_{0}=\tilde{h}(z)-\sum z_{i} u_{i}$. We clearly have $\left|\left(u_{1}, \ldots, u_{n}\right)\right|<\alpha$, and $\left|u_{0}\right|<2 \alpha$, so that $|u|<3 \alpha$. On the other hand, if we consider the function $\hat{h}=h-\left(w_{0}+u_{0}\right)-\sum\left(w_{i}+u_{i}\right) z_{i}$, then by construction $\hat{h}(z)=0$ and $\langle v, \partial \hat{h}(z)\rangle=0$. Therefore $w+u \in S$, and so $w$ is within distance $3 \alpha$ of $S$.

\section{References.}

[1] D. Auroux, Asymptotically holomorphic families of symplectic submanifolds, Geom. Funct. Anal., 7 (1997), 971-995.

[2] D. Auroux, Symplectic 4-manifolds as branched coverings of $\mathbb{C P}^{2}$, Invent. Math., 139 (2000), 551-602. 
[3] D. Auroux, Estimated transversality in symplectic geometry and projective maps, in "Symplectic Geometry and Mirror Symmetry", Proc. 4th KIAS International Conference, Seoul (2000), World Scientific, 2001, 130 (math.SG/0010052).

[4] S.K. Donaldson, Symplectic submanifolds and almost-complex geometry, J. Diff. Geom., 44 (1996), 666-705.

[5] S.K. Donaldson, Lefschetz pencils on symplectic manifolds, J. Diff. Geom., 53 (1999), 205-236.

[6] Y. Yomdin, The geometry of critical and near-critical values of differentiable mappings, Math. Annalen, 264 (1983), 495-515.

Department of Mathematics

MIT, CAMBRIDGE, MA 02139

E-mail address: auroux@math.mit.edu 